\title{
POLYARTHRITIS IN SARS-COV-2-ASSOCIATED MULTISYSTEM INFLAMMATORY SYNDROME IN ADULTS (MIS-A): A CASE REPORT
}

Renata Vaz de Oliveira ${ }^{1},{ }^{\star}$, Carlos Augusto Rodrigues Padilha ${ }^{1}$, Danielly Dantas Pimentel ${ }^{1}$, Gustavo Roberto Lourenço ${ }^{1}$, lane Tamara Dondé ${ }^{1}$, Juliana de Jesus Boscolo ${ }^{1}$, Maria Juliana da Silva Almeida ${ }^{1}$, Patrícia Milani de Moraes ${ }^{1}$, Taísa Morete da Silva

1.Faculdade de Medicina de São José do Rio Preto, São José do Rio Preto (SP), Brazil.

*Corresponding author: renata.vazdeoliveira@gmail.com

\section{BACKGROUND}

Multisystemic inflammatory syndrome (MIS) associated with SARS-CoV-2 infection is a rare complication characterized primarily in children (MIS-C); however, it has been detected in adults (MIS-A) since June 2020. MIS-A is defined on the basis of the following five criteria: presence of a severe illness in patients aged $\geq 21$ years requiring hospitalization; a positive test result for current or previous SARS-CoV-2 infection during admission or in the previous 12 weeks; presence of extrapulmonary organ dysfunction (shock, cardiac dysfunction, thromboembolic events or acute liver injury); laboratory evidence of inflammation and absence of severe respiratory disease. Furthermore, MIS-A can present with gastrointestinal and neurological symptoms, rashes, mucositis and arthralgia or arthritis remain poorly documented.

\section{CASE REPORT}

A 38-year-old Caucasian male with a history of headache and myalgia in whom SARS-CoV-2 infection was confirmed via reverse transcription polymerase chain reaction (PCR) testing. Fifteen days after testing positive, the patient was hospitalized due to the complaint of weakness, symmetric polyarthritis (shoulders, elbows and knees), and fever (temperature up to $38^{\circ} \mathrm{C}$ ) for more than 7 days, with no clinical respiratory symptoms. He exhibited no severe organ dysfunction and did not require intensive care support. Physical examination showed oral mucositis and lower limb maculopapular rash. Laboratory test results showed increased serum levels of inflammatory markers including C-reactive protein $(11 \mathrm{mg} / \mathrm{dL})$ and ferritin $(824 \mathrm{ng} / \mathrm{dL})$ and altered D-dimer levels $(2.5 \mathrm{ug} / \mathrm{mL}$ ). Chest computed tomography showed a mild "crazy paving" pattern in perihilar lung fields. Transthoracic and transesophageal echocardiogram showed no vegetation or ventricular dysfunction; furthermore, blood culture results showed no pathological microorganism growth. The patient exhibited no fever resolution even after antibiotic administration. Lower limb Doppler ultrasonography showed deep gastrocnemius and soleal vein thromboses in the left leg; therefore, anticoagulation therapy was initiated. Antiphospholipid antibody, antinuclear antibody and rheumatoid factor tests were requested to aid differential diagnosis; all tests were negative. Thus, corticotherapy was initiated (prednisone $1 \mathrm{mg} / \mathrm{kg}$ ) with the suspicion of MIS-A, resulting in complete improvement of polyarthritis and resolution of fever.

\section{CONCLUSION}

MIS-A may have a heterogeneous clinical presentation, often with a disease severity lower than that described previously, and may remain underdiagnosed. Therefore, case definitions should be constantly reviewed and MIS-A should be considered in the differential diagnosis of conditions presenting with aforementioned symptoms, including rheumatological diseases. Further research is warranted to understand the long-term effects of MIS-A and to improve its therapy.

\section{KEYWORDS}

MIS-A, SARS-CoV-2, Polyarthritis. 